

\title{
Automatic Camera-Based Microscope Calibration for a Telemicromanipulation System Using a Virtual Pattern
}

Mehdi Ammi, Vincent Fremont, Antoine Ferreira

\section{To cite this version:}

Mehdi Ammi, Vincent Fremont, Antoine Ferreira. Automatic Camera-Based Microscope Calibration for a Telemicromanipulation System Using a Virtual Pattern. IEEE Transactions on Robotics, 2009, 25 (1), pp.184-191. hal-00439818

\section{HAL Id: hal-00439818 https://hal.science/hal-00439818}

Submitted on 8 Dec 2009

HAL is a multi-disciplinary open access archive for the deposit and dissemination of scientific research documents, whether they are published or not. The documents may come from teaching and research institutions in France or abroad, or from public or private research centers.
L'archive ouverte pluridisciplinaire HAL, est destinée au dépôt et à la diffusion de documents scientifiques de niveau recherche, publiés ou non, émanant des établissements d'enseignement et de recherche français ou étrangers, des laboratoires publics ou privés. 


\title{
Automatic Camera-based Microscope Calibration for a Tele-Micromanipulation System using a Virtual Pattern
}

\author{
Mehdi Ammi*, Vincent Frémont ${ }^{\dagger}$, Antoine Ferreira ${ }^{\ddagger}$ \\ *LIMSI-CNRS, Université de Paris Sud, France \\ Email: mehdi.ammi@limsi.fr \\ ${ }^{\dagger}$ Heudiasyc-CNRS, Université de Technologie de Compiégne, France \\ Email: vincent.fremont@hds.utc.fr \\ ${ }_{\ddagger}^{\ddagger}$ Laboratoire Vision et Robotique \\ ENSI de Bourges - Université d'Orléans, France \\ Email: antoine.ferreira@ensi-bourges.fr
}

\begin{abstract}
In the context of virtualized-reality-based telemicromanipulation, this paper presents a visual calibration technique for an optical microscope coupled to a CCD camera. The accuracy and flexibility of the proposed automatic virtual calibration method, based on Parallel Single-Plane properties, are outlined. In contrast to standard approaches, a 3D virtual calibration pattern is constructed using the micromanipulator tip with subpixel-order localization in the image frame. The proposed procedure leads to a linear system whose solution provides directly both the intrinsic and extrinsic parameters of the geometrical model. Computer simulations and real data have been used to test the proposed technique, and promising results have been obtained. Based on the proposed calibration techniques, a 3D virtual microenvironment of the workspace is reconstructed through the real-time imaging of two perpendicular optical microscopes. Our method provides a flexible, easy-touse technical alternative to the classical techniques used in micromanipulation systems.
\end{abstract}

Index Terms - Camera/Microscope Calibration, Telemicromanipulation, Virtual Pattern, Virtual Reality

\section{INTRODUCTION}

In the field of microtechnologies, optical microscopes are the key sensor device for visually based micromanipulation systems where vision feedback is required for guidance manipulation strategies. Practical visual servoing examples can be found in a large variety of constrained and variable operating microenvironments such as vision feedback microassembly [1], vision servoing of biological injection tasks [2] or microscope-assisted guided surgical interventions [3]. Nevertheless real-time sensor feedback provided by the optical microscope is not always sufficient. As the image of the microscope is two-dimensional, it is difficult to manipulate the microobject in the 3D space because depth of field is small and the field of view is narrow. To improve micromanipulation we have proposed a new camera/microscope calibration for three-dimensional viewpoint selection [4] under virtual reality (VR). One major problem is the full registration of the 3D reconstructed world (virtual scene) and the 3D micromanipulator positions (real scene). Few works have been reported on VR navigation for tele-micromanipulation [5],[6], and previous methods did not consider the crucial stage of camera/microscope calibration in order to determine the correspondence between 3D world coordinates and 2-D optical microscope/camera coordinates.

A number of 3D micromanipulation approaches have been investigated. Systematic methods based on 3D high-precision micromanipulator position/orientation sensors to calibrate the relative $3 \mathrm{D}$ position and orientation between the tool and the optical microscope/camera have been proposed. However, calibration accuracy is highly dependent on the accuracy of micromanipulator kinematics [7] and on the measurement system used [8]. Moreover, calibration accuracy may deteriorate as a result of thermal deformation, vibration and various positioning errors [9].

In classical camera calibration methods [10],[11] a calibration pattern is used where the geometry of the $3 \mathrm{D}$ space is known with high precision. At a micro scale it is difficult to find a reliable micrometer-sized 3D calibration structure containing nanomarkers that serve as well-distinguishable reference points for the calibration of large 3D depths of field. Such a structure must include a 3D pattern with textures (matching is based on correlation techniques), non-repetitive motifs (to avoid ambiguity problems during matching) and features occurring at different depths (error-prone 3D reconstruction). Different microfabricated calibration patterns can be used: by printing arrays of black dots uniformly distributed over a microscopic area [12], by using engraving techniques based on optical lithography [16],[14], or by using a water drop covered with nickel filings [15]. These systems allow very high-precision patterning, but lack operational flexibility, since the calibration target must be painstakingly installed/removed with each change in magnification (modification of the optical tube lens length or optical objective). Furthermore, reattaching the micromanipulator can lead to positional errors, and attention must be paid to thermal deformations.

A promising solution is to construct 3D virtual calibration patterns automatically, using the micromanipulator end- 
effector positions as reference points. At the microscale, Sitti et al. [17] were the first to propose a 2-D automatic AFM tip center position calibration using tapping mode AFM imaging. As extension of this method, a 3D calibration technique using a glass pipette tip has been proposed by Kawaji et al. [18] where the manipulator tip is calibrated to a sub-micron order of accuracy by illuminating it with an optical fiber. However, the accuracy and robustness of pipette tip detection is greatly influenced by the microscope's lighting environment.

In this study we construct a $3 \mathrm{D}$ virtual target by positioning a piezoresistive Atomic Force Microscope cantilever (termed hereafter AFM-tip) with its tip used as end-effector and force sensor. An optical microscope coupled to a CCD camera is used as the vision sensor. The AFM-tip is connected to the micromanipulator and is randomly positioned within the $3 \mathrm{D}$ working area of the observation frame in the microscope's focal plane. The successive measurements of precise 3D positions of the AFM-tip in the current view constitutes a virtual calibration pattern. The main contribution of this paper, which is a deeper study of the work presented in [19], is to compare the calibration algorithm and its experimental evaluation with Tsai's well-known existing algorithms [10]. Moreover, since only single-plane calibration methods can be used, we propose a modified version of Zhang's algorithm [11] which yields both intrinsic and extrinsic parameters in one computational step using the micromanipulator tip center positions. Our method avoids the singularities in the frontoparallel optical microscope configuration that are a feature of Tsai's algorithms.

The remainder of this paper is as follows. In Section II we present the microscope calibration framework. Section III introduces the proposed 3D calibration methodology based on a modified Zhang's algorithm combined with the virtual pattern. Section IV provides experimental calibration results applied to the 3D reconstruction of a micromanipulation task. Finally, Section V discusses the virtual calibration performances compared to the main existing camera/microscope optical calibration methods.

\section{Microscope CALibration Framework}

\section{A. Microscope/Camera Optical Model}

Modern research microscopes are usually equipped with infinity-corrected objectives that avoid projecting the intermediate image directly onto the intermediate image plane [12]. Light emerging from these objectives is focused to infinity, and a second lens, known as a tube lens, creates the image at its focal plane and projects it onto the sensor plane (a CCD array) where a real image is formed. For the purposes of geometrical calibration, the microscope model is similar to the pinholecamera model [20] as shown in Fig.1.

A $3 \mathrm{D}$ point in the frame $R_{w}$ is defined by the coordinate vector ${ }^{\left(R_{w}\right)} \boldsymbol{M}=[X, Y, Z]^{T}$. In $R_{0}$, the $3 \mathrm{D}$ point ${ }^{\left(R_{0}\right)} \boldsymbol{M}=$ $\left[X_{0}, Y_{0}, Z_{0}\right]^{T}$ is defined by

$$
{ }^{\left(R_{0}\right)} \boldsymbol{M}=\mathbf{R} \cdot{ }^{\left(R_{w}\right)} \boldsymbol{M}+\boldsymbol{t}
$$

with

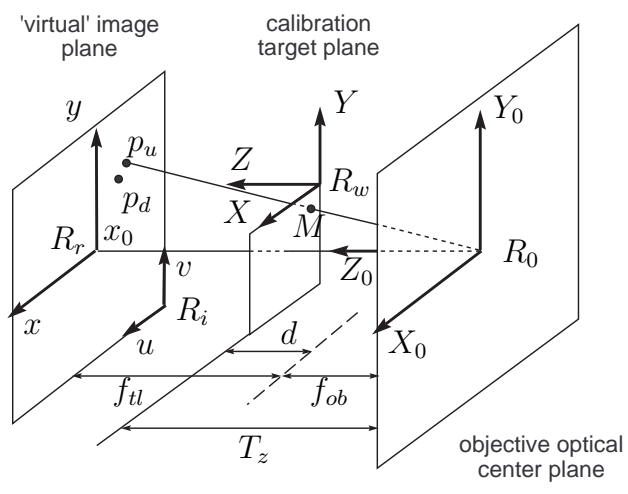

Fig. 1. Microscope pinhole model

$$
\mathbf{R}=\left[\begin{array}{lll}
r_{11} & r_{12} & r_{13} \\
r_{21} & r_{22} & r_{23} \\
r_{31} & r_{32} & r_{33}
\end{array}\right] \quad \text { and } \boldsymbol{t}=\left[\begin{array}{c}
T_{x} \\
T_{y} \\
T_{z}
\end{array}\right]
$$

$\mathbf{R}$ is a rotation matrix with three degrees of freedom, and $\boldsymbol{t}$ is a translation vector also with three degrees of freedom. These six degrees of freedom are known as the extrinsic parameters of the microscope/camera optical model.

We use the notation $\widetilde{x}$ to denote a vector in homogenous coordinates, i.e. if $x=[a, b]^{T}$ then $\widetilde{x}=[a, b, 1]^{T}$.

By perspective projection, the relationship between ${ }^{\left(R_{0}\right)} \boldsymbol{M}$ and the image point ${ }^{\left(R_{i}\right)} \widetilde{\boldsymbol{p}}_{u}=[u, v, 1]^{T}$ in $R_{i}$ is

$$
s\left[\begin{array}{l}
u \\
v \\
1
\end{array}\right]=\left[\begin{array}{ccc}
k_{u} f_{\text {tot }} & s_{k} & u_{0} \\
0 & k_{v} f_{\text {tot }} & v_{0} \\
0 & 0 & 1
\end{array}\right]\left[\begin{array}{c}
X_{0} \\
Y_{0} \\
Z_{0}
\end{array}\right]
$$

where $s$ is an arbitrary scale factor and $f_{t o t}=f_{t l}+f_{o b}$. The parameters $k_{u}, k_{v}, s_{k}, u_{0}, v_{0}, f_{t l}$ and $f_{o b}$ are known as the intrinsic parameters of the microscope/camera optical model and represent respectively the two projection gains, the skewness, the coordinates of the principal point, the optical tube length and the exact focal length. Taking into account the radial lens distorsion of the microscope/camera system and its center as the principal point, the distorted image point ${ }^{\left(R_{i}\right)} \widetilde{\boldsymbol{p}}_{d}=\left[u_{d}, v_{d}, 1\right]^{T}$ can be expressed as

$$
\begin{aligned}
& u_{d}=u+\left(u-u_{0}\right)\left(k_{1} r^{2}+k_{2} r^{4}\right) \\
& v_{d}=v+\left(v-v_{0}\right)\left(k_{1} r^{2}+k_{2} r^{4}\right)
\end{aligned}
$$

where $k_{1}$ and $k_{2}$ are the coefficients of the radial distorsion and $r^{2}=u_{n}^{2}+v_{n}^{2}$ with $\left(u_{n}, v_{n}\right)$ the normalized distortion-free image coordinates.

The main constraint in the optical microscopy calibration is the low depth of field of the objectives. This implies that multiple parallel calibration planes perpendicular to the optical axis and located at varying depths cannot be applied in microscope calibration. Moreover, rotating the calibration pattern relative to the image plane will result in a blurred image, making accurate geometry extraction impossible. Since the calibration plane cannot be exactly parallel to the image plane, we consider a near-parallel approximation $(\cos x \cong 1$, $\sin x \cong x$ ) leading to the following simplified rotation matrix 


$$
\left[\begin{array}{lll}
r_{11} & r_{12} & r_{13} \\
r_{21} & r_{22} & r_{23} \\
r_{31} & r_{32} & r_{33}
\end{array}\right]=\left[\begin{array}{ccc}
c \alpha & -s \alpha & c \alpha \beta+s \alpha \gamma \\
s \alpha & c \alpha & s \alpha \beta-c \alpha \gamma \\
-\beta & \gamma & 1
\end{array}\right]
$$

where $c x \equiv \cos x$ and $\alpha, \beta, \gamma$ represent the Euler angles. Finally, the reduced parametric microscope/camera optical model is

$$
s\left[\begin{array}{l}
u \\
v \\
1
\end{array}\right]=\mathbf{A}\left[\begin{array}{ccc}
c \alpha & -s \alpha & T_{x} \\
s \alpha & c \alpha & T_{y} \\
-\beta & \gamma & T_{z}
\end{array}\right]\left[\begin{array}{c}
X \\
Y \\
1
\end{array}\right]
$$

where

$$
\mathbf{A}=\left[\begin{array}{ccc}
f_{1} & s_{k} & u_{0} \\
0 & f_{2} & v_{0} \\
0 & 0 & 1
\end{array}\right]
$$

with $f_{1}=k_{u}\left(f_{t l}+f_{o b}\right)$ and $f_{2}=k_{v}\left(f_{t l}+f_{o b}\right)$. The magnification of the microscope/camera system is defined as

$$
M=\frac{f_{o b}+f_{t l}}{T_{z}}
$$

\section{B. High-Accuracy Target Construction}

In addition to the optical constraint, tele-micromanipulation systems are generally characterized by limited manipulation space. The use of the conventional calibration target (e.g., printed by lithography pattern) seems impractical, since the pattern must be manipulated within a confined space before and after each calibration step. As stated in the introduction, in order to realize accurate measurements and to achieve good calibration results, particular care has to be taken in the way the calibration target is constructed, and also in the way the coordinates of calibration points are measured. Since using a conventional calibration target is not practical in the micromanipulation case, we have chosen to carry out the calibration pattern with the micromanipulator. The target (AFM-tip) was mounted on the micromanipulator's endeffector whose position is controlled by the calibration module [4]. The role of this component is to generate successive positions to construct the virtual target by taking into account the various objects (microspheres and dust particles) which constitute the manipulated scene. The generated positions must be:

- in the same plane, because of the optical constraints;

- randomly positioned in the scene to guarantee the independence of the nonlinear equations involved in the calibration algorithm.

The pattern construction procedure is as follows:

1) the image processing module first locates the various objects present in the scene ;

2) the configuration-space (CS) of the manipulated scene is then computed according to the geometry of the obstacles and the AFM-tip ;

3) finally, the calibration module generates several random positions in the CS-free (see Fig. 2).

The spatial position of the AFM-tip is determined from the position of the micromanipulator on the assumption that the $x-y-z$ piezoelectric positioning stage is very precise (closedloop control) with a nanometer positioning accuracy.

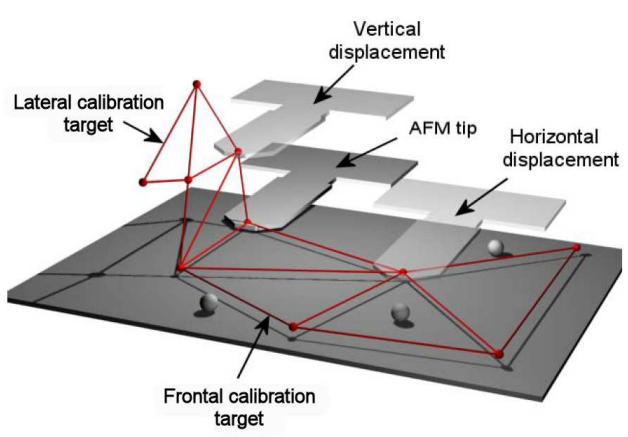

Fig. 2. Schematic calibration target construction using localization of the AFM-tip positions.

In order to recover the position of the AFM-tip with high accuracy, we choose the image normalized correlation technique [20] which is fast, invariant to linear radiometric changes and can be implemented in a context of Kalman filtering (Gaussian noise assumptions) [23] or particle filtering (nonGaussian noise assumptions) [24]. Methods such as wavelet decomposition may be used to improve the tracking precision with a multi-resolution and auto-focusing approach [25]. The approach we adopted uses a reference template of image data $M(u, v)$ and a test image $I(m, n)$. The correlation is carried out by comparing a template image to all sub-parts of the corresponding image region at the pixel level. The correlation represents the similarity between the pattern and the corresponding region. The point where the correlation reaches a local minimum value is considered to be the matching target point. As stated earlier, in order to make the correlation insensitive to global luminosity variations the Normalized Correlation Coefficient [20] is used:

$$
N C C=\frac{\sum_{u, v} M(u, v) \cdot I(m+u, n+v)}{\sqrt{\left|\sum_{u, v} M^{2}(u, v) \cdot \sum_{u, v} I^{2}(m+u, n+v)\right|}} .
$$

The similarity measurement is robust to partial visual object occlusions and in practice provides stable detection results [26]. Using the correlation approach to locate a target instance, the locations $m$ and $n$ return integer values. Assuming that the correlation surface is relatively smooth, it is possible to interpolate between sample values to estimate a subpixel localization of the target. We propose a method using a threedimensional paraboloid surface that fits the set of normalized correlation data points. Once the integral location is found, the eight neighbors on the correlation surface are also computed in order to fit $z(m, n)$ a generalized equation defined by

$$
z=a . m^{2}+b \cdot n^{2}+c \cdot m \cdot n+d \cdot m+e \cdot n+f .
$$

For several image points $\left(m_{i}, n_{i}\right)$, and stacking all equations (with a minimum of six points) expressed by (9), we obtain a linear system

$$
\mathrm{Zm}=\boldsymbol{b} .
$$


The closed-form solution of (10) can be obtained by a leastsquares regression method using a singular value decomposition (SVD) [27] of $\mathbf{Z}$. Once the coefficients of the surface are known, the maximum of the paraboloid surface function gives the optimal subpixel coordinates. This algorithm has the advantage of achieving high accuracy without the need for large magnification optics.

\section{Proposed Calibration Methodology}

As stated before, optical principles dictate that for optical microscopes, the object plane must be parallel or very nearly parallel to the image plane. However, existing methods do not work well with calibration patterns that are parallel to the CCD sensor. For example, Tsai's algorithm [10],[28] requires that the calibration pattern has to be tilted at least 30 degrees relative to the image plane. In the parallel case this algorithm is singular [22]. To take this into account, the basic idea is to simplify the entire calibration model by assuming small angle approximations to the pitch and yaw angles of the rotation matrix. When these angles become larger, the error due to the linear approximation becomes more significant. On the other hand, methods based on several planar calibration patterns like Zhang's algorithm use the concept of the Homography transformation [11],[29]. Single image calibration is possible, but it reduces the number of intrinsic parameters to be solved. In this section, a calibration algorithm based on Zhang's procedure [11], modified for the parallel case, is proposed for optical microscope calibration.

\section{A. Proposed Modification to Zhang's Method}

Starting from (5), an object point is related to the corresponding image point by a homography $\mathbf{H}$, defined as

$$
s .^{\left(R_{i}\right)} \widetilde{\boldsymbol{p}}_{u}=\mathbf{H} \cdot{ }^{\left(R_{w}\right)} \widetilde{\boldsymbol{M}} \text { with } \mathbf{H}=\mathbf{A}\left[\begin{array}{lll}
\boldsymbol{r}_{1} & \boldsymbol{r}_{2} & \boldsymbol{t}
\end{array}\right] .
$$

where $\boldsymbol{r}_{i}$ is the $i^{\text {th }}$ column of the rotation matrix $\mathbf{R}$.

The homography is a linear transformation which maps the image plane onto the world object plane, and it is clear that the $3 \times 3$ matrix $\mathbf{H}$ is defined up to a scale factor.

The two projection gains are defined as follows

$$
k_{u}=\frac{s_{x} \cdot N_{f x}}{d_{x} \cdot N_{c x}} \quad \text { and } \quad k_{v}=d y
$$

where $d_{x}$ and $d_{y}$ are the effective center-to-center distances between the camera's sensor elements in the $u$ and $v$ directions; $N_{c x}$ is the number of sensor elements in the $u$ direction; $N_{f x}$ is the number of pixels in a line as sampled by computer and $s_{x}$ is the uncertainty image scale factor. The term $s_{x}$ is defined as the ratio between the aspect ratio of the sensing area and the aspect ratio of the frame buffer. It can be expressed as follows

$$
s_{x}=\left(\frac{C C D_{x}}{C C D_{y}}\right) /\left(\frac{N_{f x}}{N_{f y}}\right)
$$

where $C C D_{x}$ and $C C D_{y}$ are the dimensions of the sensing area in the $u$ and $v$ directions, and $N_{f y}$ is the number of pixels on the frame grabber in the $v$ direction.
1) Recovering intrinsic parameters : As stated earlier, $\mathbf{H}$ is defined up to a scale factor. This homography between the object plane and the image plane can be estimated using the $3 \mathrm{D}$ coordinates of the micromanipulator and the corresponding image points. A minimum of six points are necessary to obtain a solution [30]. A random sampling and consensus (RANSAC) algorithm [31] is used to eliminate outlying data points that come from tracking errors and contaminate the estimation process.

Let $\boldsymbol{h}_{i}$ be the $i^{\text {th }}$ column in H. From (11), we have:

$$
\lambda\left[\begin{array}{lll}
\boldsymbol{h}_{1} & \boldsymbol{h}_{2} & \boldsymbol{h}_{3}
\end{array}\right]=\mathbf{A}\left[\begin{array}{lll}
\boldsymbol{r}_{1} & \boldsymbol{r}_{2} & \boldsymbol{t}
\end{array}\right] .
$$

where $\lambda$ is an arbitrary scalar. The matrix $\mathbf{B}=\mathbf{A}^{-\mathbf{T}} \mathbf{A}^{-1}$ represents the image of the absolute conic [32]. From only one image, we can solve both intrinsic parameters $f_{1}$ and $f_{2}$ (imposing the skew constraints $s_{k}=0$, and assuming $u_{0}$ and $v_{0}$ are known and located in the middle of the image and are therefore image points expressed in $\left(R_{r}\right)$ ). ¿From (14), we also have $\boldsymbol{r}_{1}=\lambda \mathbf{A}^{-1} \boldsymbol{h}_{1}$ and $\boldsymbol{r}_{2}=\lambda \mathbf{A}^{-1} \boldsymbol{h}_{2}$. Consequently we obtain

$$
\begin{aligned}
& \boldsymbol{r}_{1}^{T} \boldsymbol{r}_{1}=\lambda^{2} \boldsymbol{h}_{1}^{T} \mathbf{B} \boldsymbol{h}_{1}=1 \\
& \boldsymbol{r}_{2}^{T} \boldsymbol{r}_{2}=\lambda^{2} \boldsymbol{h}_{2}^{T} \mathbf{B} \boldsymbol{h}_{2}=1 \\
& \boldsymbol{r}_{1}^{T} \boldsymbol{r}_{2}=\lambda^{2} \boldsymbol{h}_{1}^{T} \mathbf{B} \boldsymbol{h}_{2}=0 .
\end{aligned}
$$

Since $\boldsymbol{r}_{1}$ and $\boldsymbol{r}_{2}$ are orthonormal, we have:

$$
\boldsymbol{h}_{1}^{T} \mathbf{B} \boldsymbol{h}_{2}=\boldsymbol{h}_{1}^{T} \mathbf{B} \boldsymbol{h}_{1}-\boldsymbol{h}_{2}^{T} \mathbf{B} \boldsymbol{h}_{2}=0 .
$$

These relations allow us to solve only two parameters $\left(f_{1}\right.$ and $f_{2}$ ); in order to recover the parameter $\lambda$ it is necessary to add another constraint. From (11), we can introduce an additional constraint expressed as

$$
\lambda=\frac{1}{\left\|\mathbf{A}^{-1} \boldsymbol{h}_{1}\right\|} .
$$

After some developments, we obtain the following expression

$$
\lambda=1 / \sqrt{\left(h_{11} / f_{1}\right)^{2}+\left(h_{21} / f_{2}\right)^{2}+h_{31}^{2}}
$$

where $h_{i j}$ are line-column elements of the matrix $\mathbf{H}$.

Finally, the previous equation can be written as follows:

$$
\frac{h_{11}^{2}}{M_{1}{ }^{2}}+\frac{h_{21}^{2}}{M_{2}^{2}}+h_{31}^{2} \lambda^{2}=1
$$

where $M_{1}=\frac{f_{1}}{\lambda}$ and $M_{2}=\frac{f_{2}}{\lambda}$ are the magnifications corresponding respectively to the $u$ and $v$ axes.

By adding this constraint to (18), we obtain a constrained system enabling us to recover the two magnification parameters $M_{1}$ and $M_{2}$, along with the scale factor $\lambda$. The final system can be expressed as follows:

$$
\left[\begin{array}{ccc}
h_{11} h_{12} & h_{21} h_{22} & h_{31} h_{32} \\
h_{11}^{2}-h_{12}^{2} & h_{21}^{2}-h_{22}^{2} & h_{31}^{2}-h_{32}^{2} \\
h_{11}^{2} & h_{21}^{2} & h_{31}^{2}
\end{array}\right]\left[\begin{array}{c}
\frac{1}{M_{1}{ }^{2}} \\
\frac{1}{M_{2}^{2}} \\
\lambda^{2}
\end{array}\right]=\left[\begin{array}{l}
0 \\
0 \\
1
\end{array}\right]
$$


¿From (7) the total focal length of the tube lens and the total focal length of microscope can be extracted:

$$
f_{t l}=M_{1} \cdot f_{o b}=s_{x} \cdot M_{2} \cdot f_{o b}
$$

2) Recovering extrinsic parameters: Once $\mathbf{A}$ is known, the extrinsic parameters for each image can be computed. From (11), we have:

$$
\begin{aligned}
\alpha & =\arctan \left[\left(M_{1} h_{21}\right) /\left(M_{2} h_{11}\right)\right] \\
\beta & =-\lambda h_{31} \\
\gamma & =\lambda h_{32} \\
T_{x} & =h_{13} / M_{1} \\
T_{y} & =h_{12} / M_{2} \\
T_{z} & =f_{o b} \frac{M+1}{M}
\end{aligned}
$$

where $M=\left(M_{1}+M_{2}\right) / 2$ and $h_{i j}$ is the element of $\mathbf{H}$ located at the $i^{\text {th }}$ line and at the $j^{\text {th }}$ column of the matrix.

3) Recovering distortion parameters: The strategy for estimating $k_{1}$ and $k_{2}$ is to calculate them after recovering the other parameters using the techniques described above, which gives the ideal pixel coordinates $(u, v)$. Eq. (3) can be set as a linear system as proposed in [11] to estimate $k_{1}$ and $k_{2}$. Finally, a nonlinear refinement through maximum likelihood inference is done using the Levenberg-Marquardt Algorithm [33] on the following nonlinear criterion:

$$
J=\min _{\Omega} \sum_{i=1}^{N}\left[\left(u_{i m}-u_{d}\right)^{2}+\left(v_{i m}-v_{d}\right)^{2}\right]
$$

with $\Omega=\left[k_{u}, k_{v}, f_{t o t}, \lambda, u_{0}, v_{0}, k_{1}, k_{2}\right]$ and $u_{i m}, v_{i m}$ the real (observed) image coordinates.

\section{EXPERIMENTAL RESULTS}

Several tests have been carried out in order to measure the accuracy of the proposed technique. These tests are based on camera/microscope simulations and experimental data. A comparison with Tsai's basic technique [10],[12] is also presented in order to evaluate the gain in accuracy.

\section{A. Noise Robustness}

The simulated camera has the following properties: $u_{0}=$ $384, v_{0}=288$, image resolution $768 \times 576$ pixels, the microscope has an objective focal length of $f_{o b}=100000 \mu \mathrm{m}$ and an optical tube focal length of $f_{t l}=200000 \mu \mathrm{m}$. The calibration target consists of a set of 100 points randomly positioned along the calibration plane nearly parallel to the CCD sensor. Gaussian noise with zero mean error and $\sigma$ standard deviation (noise level) is added to the projected points. For each noise level, this operation is performed a hundred times, and the average is taken as the result.

Fig. 3 represents the mean reprojection error with respect to the noise level. It is straightforward that our algorithm gives better results than Tsai's method. Similar results can be seen in Fig. 4 for several numbers of points in the pattern. However, the curves tend to fuse together when considering a large number of points ( $>1000$ points), but it would be unrealistic, from a practical point of view, to rely on this assumption. In practice, we choose a minimum of 24 calibration points for noise robustness evaluation, as shown in Fig. 5, 6, 7, 8, and . The estimation of the magnification (Fig. 5) and $T_{z}$ (Fig. 8) gives better results than Tsai's method. The explanation for this lies in our assumption that the object plane and image plane are nearly parallel. In this case the homography transformation performs well, since the magnification and $T_{z}$ are dominant parameters. On the other hand, $T_{x}$ (Fig. 6) and $T_{y}$ (Fig. 7), defined as small parameters, are both estimated in the same way. It will also be noticed that $\alpha$ is better estimated using our algorithm (Fig. ), since the other angles are neglected.

\section{B. Tests under real experimental conditions}

Our experimental setup is shown in Fig. 11. Key components of the setup include the following.

- Frontal microscope: Mitutoyo FS70Z with three objectives mounted on a Wentworth probe station [21].

- CCD camera: Chugai Boyeki FC-55-II module connected to a Sony sensor with 500 by 582 sensing elements and cell size of 9.8 by $6.3 \mu \mathrm{m}$;

- Lateral microscope: A TIMM-150 microscope with magnification range from " 0.1 " to " 150 ". The CCD camera is integrated and has the following characteristics: 540 by 600 sensor elements and cell size of 7.3 by $4.7 \mu \mathrm{m}$;

- Frame grabber: A mvDELTA Matrix vision frame grabber, with 768 by 576 pixels and pixel frequency of 14.750 $\mathrm{MHz}$ (to obtain square pixels);

- Micromanipulator: A M-111.1 from Polytec PI with an accuracy of $0.05 \mu \mathrm{m}$ and repeatability of $0.1 \mu \mathrm{m}$ with a piezoresistive AFM-tip effector.

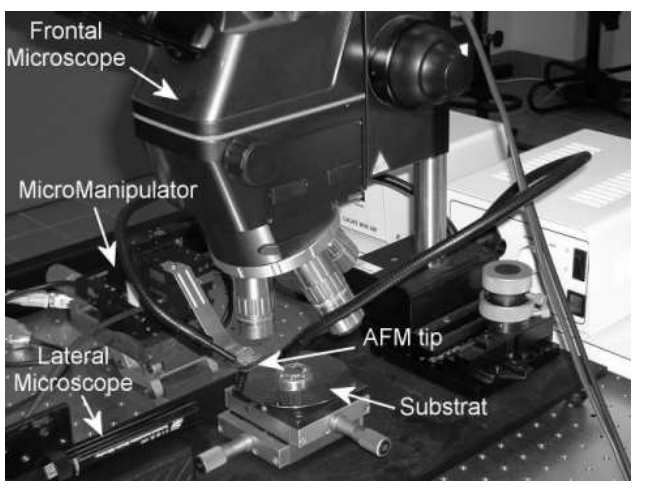

Fig. 11. Experimental setup.

The microscope calibration is a two-stage technique. The first involves generating the data for the calibration algorithms via two controller and image processing modules. The controller module generates a random sequence of end-effector positions with a precision of $0.1 \mu \mathrm{m}$ while avoiding obstacles (microspheres or dust particles). For each given position, the image processing module digitizes and computes the image in order to obtain the subpixel position of the AFM-tip. The accuracy of the subpixel estimation is $1 / 16$ of a pixel, which gives $0.6125 \mu \mathrm{m}$ in the $x$ direction and $0.3937 \mu \mathrm{m}$ in the $y$ direction. 

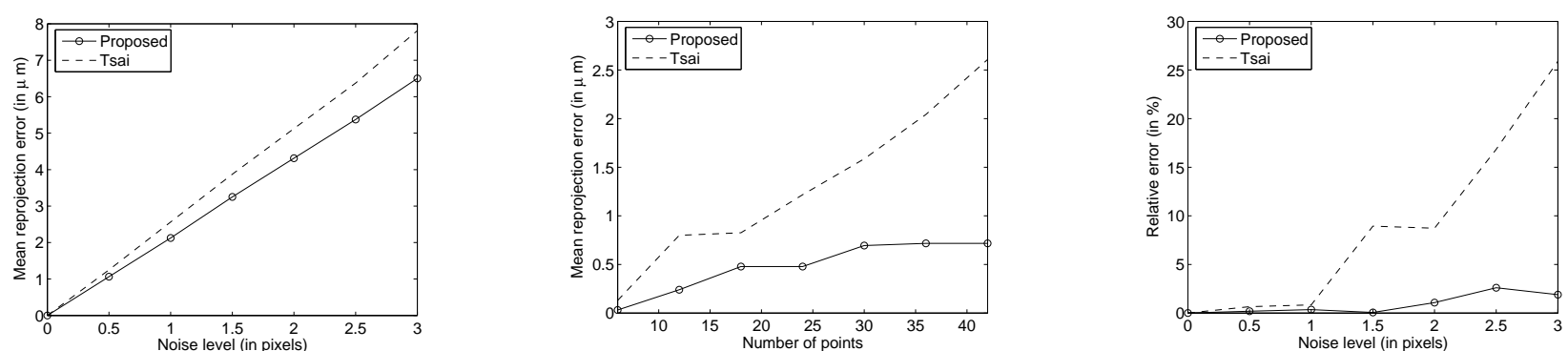

Fig. 3. Mean reprojection error with respect to noise Fig. 4. Mean reprojection error with respect to Fig. 5. Relative error on the magnification with level.

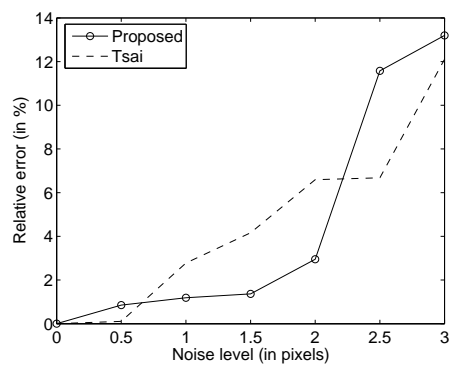
number of pattern points.

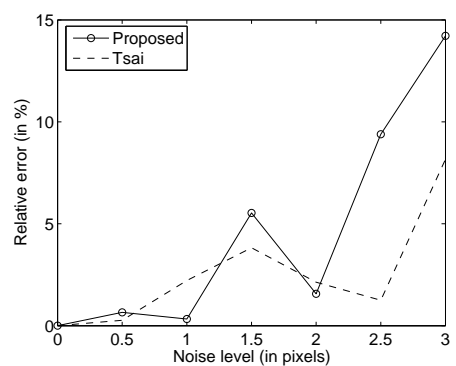
respect to noise level.

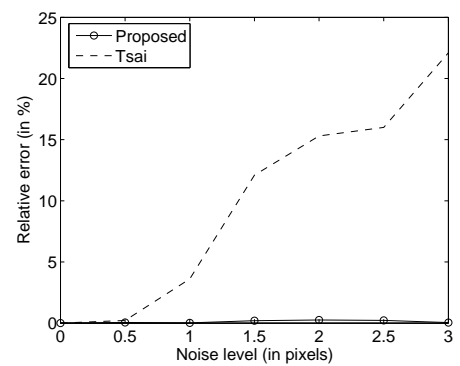

Fig. 6. Relative error on $T_{x}$ with respect to noise Fig. 7. Relative error on $T_{y}$ with respect to noise Fig. 8. Relative error on $T_{z}$ compare to noise level. level.

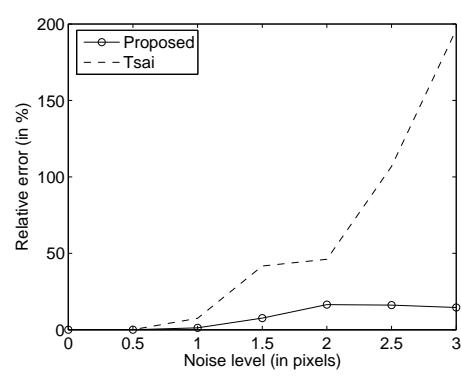

Fig. 9. Relative error on $\alpha$ with respect to noise level.

It can be seen from Table I that our proposed algorithm gives better results on the 3D reprojection error than Tsai's method. It should also be noticed that our method can estimate both intrinsic and extrinsic parameters from the homography matrix defined in (11).

\section{Execution time}

The total execution time of the calibration procedure depends mainly on the complexity of the virtual pattern construction and on the execution time of the calibration algorithm. Since the virtual pattern is constructed incrementally, the construction time is directly proportional to the number of points. The total time for one calibration point $T_{t o t}$ is defined as the summation

$$
T_{t o t}=T_{d p}+T_{d s}+T_{l c}=0.827 s
$$

where $T_{d p}$ is the displacement time, $T_{d s}$ the stabilization time and $T_{l c}$ the localization time. In practice, several tests indicate that for a number of points varying between between 6 to 50 , the computation time is approximately constant $\left(T_{t o t} \simeq\right.$ $0.8 \mathrm{~s})$.

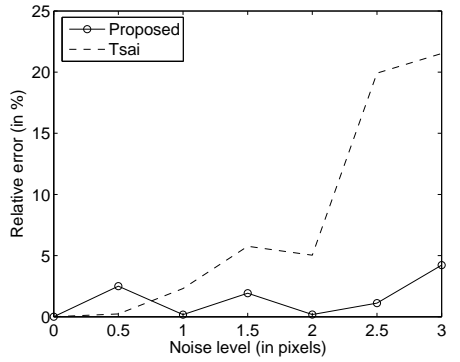

Fig. 10. Relative error on $f_{1}$ with respect to noise level.

\section{Application to $3 D$ microenvironment reconstruction for virtualized tele-micromanipulation}

The aim of microscope calibration is to allow faithful registration of a $3 \mathrm{D}$ real-time reconstruction environment for virtualized tele-micromanipulation. It formulates the virtualized reality of the microenvironment through two sources of information: (i) from real images provided by optical microscopy and (ii) from synthetic views generated by a 3D model of the remote microworld. The image scene is made up of three types of objects, i.e., substrate, microspheres and dust particles. The $3 \mathrm{D}$ reconstruction is done by the images returned from both the frontal and lateral microscopes. The main steps of 3D micro-environment reconstruction are shown in the algorithm (1). Using our calibration algorithm, we estimate the homography for each microscope: ${ }^{(F)} \mathbf{H}$ for the frontal microscope (Eq. (27)) and ${ }^{(L)} \mathbf{H}$ for the lateral microscope (Eq. (28)).

$$
s\left[\begin{array}{c}
u_{F} \\
v_{F} \\
1
\end{array}\right]=\left[\begin{array}{lll}
{ }^{(F)} h_{11} & { }^{(F)} h_{12} & { }^{(F)} h_{13} \\
(F) h_{21} & { }^{(F)} h_{22} & { }^{(F)} h_{23} \\
(F) h_{31} & (F) h_{32} & (F) h_{33}
\end{array}\right]\left[\begin{array}{c}
X \\
Y \\
1
\end{array}\right]
$$




$$
\eta\left[\begin{array}{c}
u_{L} \\
v_{L} \\
1
\end{array}\right]=\left[\begin{array}{lll}
{ }^{(L)} h_{11} & { }^{(L)} h_{12} & { }^{(L)} h_{13} \\
{ }^{(L)} h_{21} & { }^{(L)} h_{22} & (L) h_{23} \\
{ }^{(L)} h_{31} & { }^{(L)} h_{32} & { }^{(L)} h_{33}
\end{array}\right]\left[\begin{array}{c}
Y \\
Z \\
1
\end{array}\right]
$$

Each homography gives a linear mapping between the microscope image plane and its world focused plane. Combining (27) and (28) gives a linear system $\mathbf{C} \widetilde{\boldsymbol{M}}=\boldsymbol{0}$. The solution vector $\widetilde{\boldsymbol{M}}$ of the system is the right null-space of $\mathbf{C}$ which can be obtained from its Singular Value Decomposition [27], since image point coordinates are corrupted by noise. This process, known as linear triangulation, allows the spatial position of each object in the $X-Y$ plane as well as the localization of the AFM-tip in the $Y-Z$ plane of the reference frame $R_{w}$ to be retrieved. Since image coordinates are corrupted by noise, a nonlinear refinement through maximum likelihood inference can be done using the Levenberg-Marquardt Algorithm [33] by minimizing the 2-D reprojection error starting from the initial estimate of $\widetilde{\boldsymbol{M}}$. When the spheres are moved to or removed from different locations, a regular update is performed at the image processing frequency. In order to reduce the computation time, we consider that the different impurities are static in the environment. The maximum computation time, including the image processing step, the $3 \mathrm{D}$ reconstruction and the update of scene algorithms, is about $95.17 \mathrm{~ms}$ on a Pentium IV-2.66 GHz CPU with 512 Mo DDRAM on a Windows 2000 platform. The 3D graphic rendering is based on the OPEN-INVENTOR C++ graphic library on a Win32 platform; this library provides powerful functions for graphical immersion and software architecture. Once the micro-world is reconstructed, we can obtain perspective views of the virtual scene from any viewpoint (see Fig. 12).

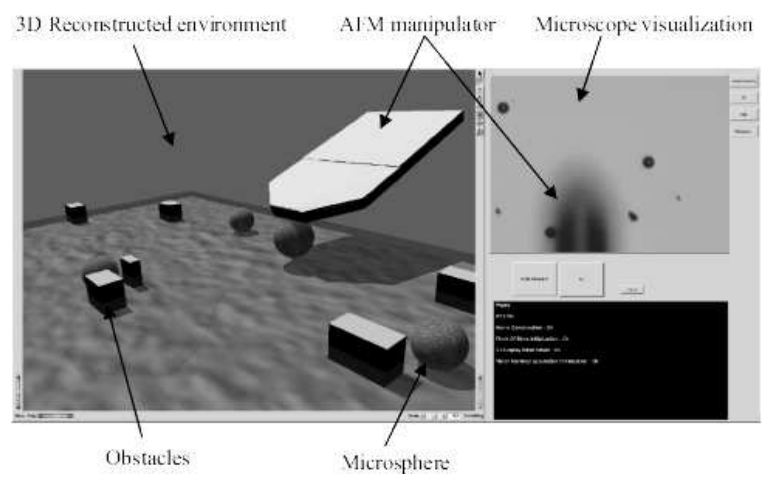

Fig. 12. Graphical user interface (GUI) integrating the real imaging (righthand side) provided by the optical microscope and the reconstructed virtual microenvironment (left-hand side) during real-time AFM micromanipulation.

\section{DISCUSSION AND CONCLUSION}

In this paper we have developed a new $3 \mathrm{D}$ flexible technique to calibrate an optical microscope mounted on a CCD camera for virtualized-reality-based tele-micromanipulation applications. Our method does not require a physical calibration pattern, but only a tracking of the micromanipulator positions and its corresponding image points. The proposed calibration algorithm is based on Zhang's method using single and parallel calibration plane constraints. It provides, in one step, both

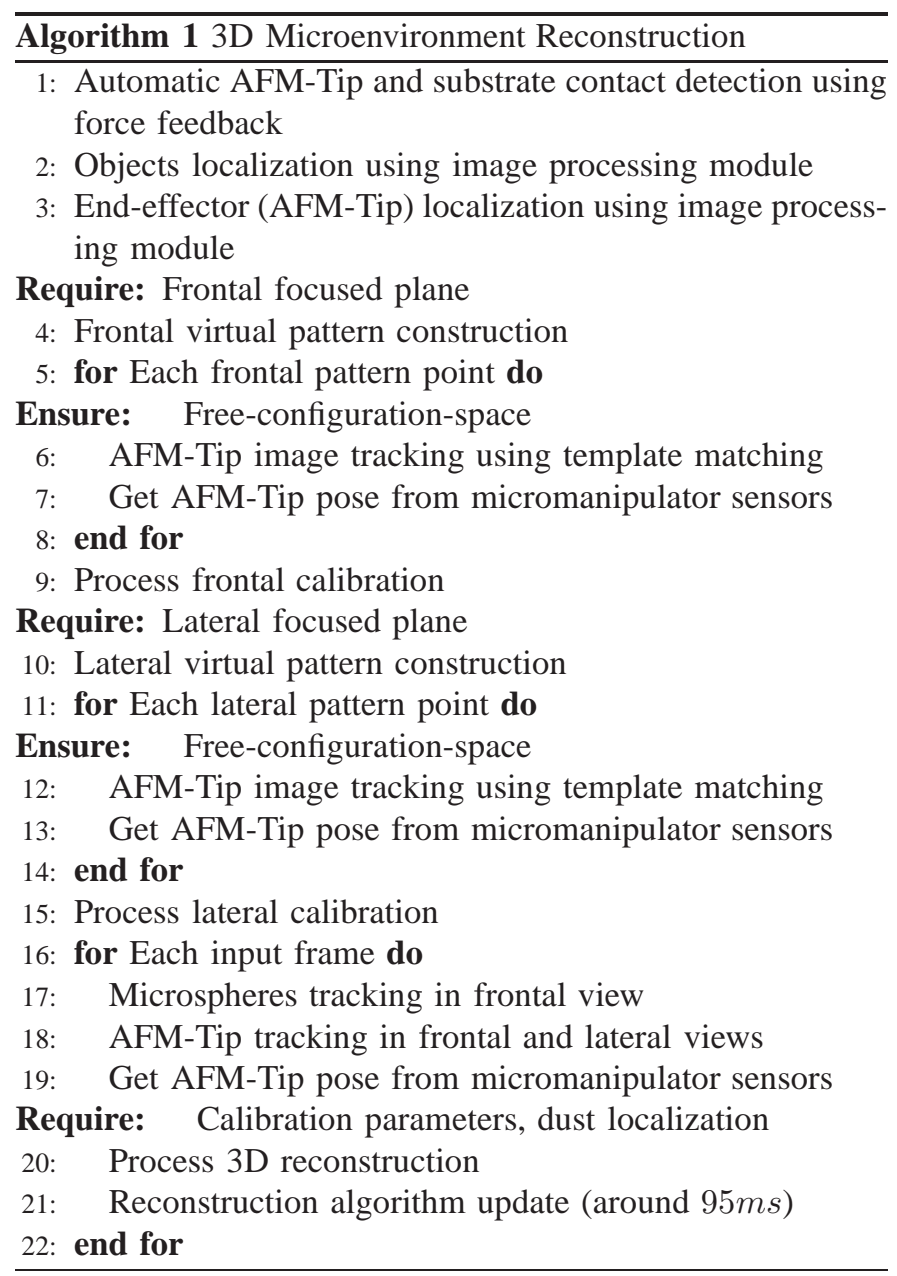

intrinsic and extrinsic parameters of the parametric camera/optical model. Both computer simulations and real data have been used to test the proposed technique, and confident results have been obtained.

Tables II-III present a comparison of some reference methods for optical camera/microscope calibration. It can be seen that most of them (the exception being a method presented in [?]) use a calibration pattern combined with Tsai's algorithm to estimate the parameters of an explicit camera/microscope model. Two main categories can be considered; methods with a microscopic real check pattern [3][?][12] and methods with a virtual calibration pattern [17][18]. The main drawback of the first category is that in the near-parallel case the iterative algorithms require a very good initial estimate of parameters, because of numerical stability problems. Moreover, relatively small amounts of noise in the data may lead to large divergence and instability (e.g. [?][12]). Nonlinear optimization algorithms should be used to achieve a stable and globally convergent iteration for intrinsic parameter determination [12] - The second category also poses a particular problem for calibration: during construction of the virtual pattern, the feature-tracking procedure is a significant source of errors and needs to be handled carefully. This has led the authors in [17] to use several hundred calibration points to minimize the $3 \mathrm{D}$ reprojection error, thus increasing considerably the overall execution time of the calibration procedure. Therefore, 
to minimize the impact of the tracking stage errors, our algorithm needs only 24 points to construct automatically the virtual pattern and to achieve very good calibration results: $0.234 \mu \mathrm{m}$ with $\mathrm{a} \times 2$ objective for the $3 \mathrm{D}$ mean reprojection error. Moreover, our virtual pattern does not need to have a regular-spaced design, and positional outliers are automatically rejected using the RANSAC procedure. Compared to [17], similar positioning precision can be achieved for higher microscope objectives; for example, a nominal positioning accuracy of $1 \mathrm{~nm}$ can be achieved with a $\times 40$ microscope objective. Nevertheless, the nonlinear least-squares error minimization in combination with with the RANSAC outliers rejection stage reduces the influence of the various positioning errors. The fronto-parallel configuration assumption seems to be a good compromise, since the yaw and pitch angles are very small and therefore roughly estimated. This assumption also speeds up the algorithm $(0.827 \mathrm{~ms}$ per pattern point $)$ and allows a direct computation of both intrinsic (including distorsion coefficients) and extrinsic parameters of the camera/microscope geometrical model. Our approach is an fast, robust, highprecision alternative to classical microscope calibration algorithms [12][17].

\section{ACKNOWLEDGMENT}

The authors would like to thank the Laboratoire de Vision et Robotique, France, for its support during the period 20022005, and all the people who were involved in this work.

\section{REFERENCES}

[1] A. Ferreira, C. Cassier, S. Hirai, Automated Microassembly System Assisted by Vision Servoing and Virtual Reality, IEEE Transactions on Mechatronics, Vol. 9, No.2, June 2004, 321-333.

[2] Y. Sun and B. J. Nelson, Microrobotic Cell Injection, IEEE International Conference on Robotics and automation, Seoul, Korea, 2001, 620-625.

[3] P. J. Edwards, et al., Design and Evaluation of a System for MicroscopeAssisted Guided Interventions, IEEE Transactions on Medical Imaging, 19(11), 2000, 1082-1093.

[4] M. Ammi, A. Ferreira, Virtualized Reality Interface for TeleMicromanipulation, IEEE International Conference on Robotics and automation, New-Orleans, USA, 2004, 2776-2781.

[5] A. Sulzman, et al., Virtual Reality and High Accurate Vision Feedback as Key Information for Micro Telemanipulation, SPIE, Microrobotics and Microassembly, vol.2906, 1990, 38-57.

[6] F. Arai, A. Kawaji, P. Luangjarmekorn, T.Fukuda, K.Itoigawa, ThreeDimensional Bio-Micromanipulation under the Microscope, IEEE International Conference on Robotics and Automation, Seoul, Korea, 2001, 601-609.

[7] S.J. Ralis; B. Vikramaditya; B.J Nelson, Micropositioning of a weakly calibrated microassembly system using coarse-to-fine visual servoing strategies. IEEE Transactions on Electronics Packaging Manufacturing, Vol.23, Issue 2, Apr 2000, 123-131.

[8] G. Li and N. Xi, Calibration of a Micromanipulation System, IEEE International Conference on Intelligent Robots ans Systems, 2002, 17421747.

[9] A. Kawaji, F. Arai, T. Fukuda, Calibration for Contact Type of Micromanipulation, IEEE International Conference on Intelligent Robotics and Systems, Vol.2, 1999, 715-720.

[10] R.Y. Tsai, A Versatile Camera Calibration Technique for High-Accuracy 3D Machine Vision Metrology Using Off-the-Shelf TV Cameras and Lenses, IEEE Transactions on Robotics and Automation, 3(4),1987, 323344

[11] Z.Y. Zhang, Flexible Camera Calibration by Viewing a Plane from Unknown Orientations, IEEE International Conference on Computer Vision, 1999, 666-673.

[12] J. Seyfried, et al., Exploring the Micro and Nano World with $\mathrm{cm}^{3}$ Sized Autonomous Micro Robots, Industrial Robot, 31(2), 2004, 159-178.
[13] Y. Zhou and B.J. Nelson, Calibration of a parametric model of an optical microscope, Optical Engineering, 38(12), 1999, 1989-1995.

[14] M. Ritter, M. Hemmleb, O. Sinram, J. Albertz, H. Hohenberg, A Versatile 3D Calibration Object for Various Micro-Range Measurements Methods, XXth ISPRS Congress, 12-23 July 2004 Istanbul, Turkey, pp.696-702.

[15] J. Bert, S. Dembl, N. Lefort-Piat, Performing Weak Calibration at the Microscale: Application to Micromanipulation, IEEE International Conference on Robotics and Automation, May 2007, Roma, Italy, 49374942.

[16] F. Sinz, J. Quionero-Candela, G. H. Bakir, C. E. Rasmussen and M.O. Franz, Learning Depth From Stereo, DAGM Pattern Recognition Symposium 2004, 2004, 245-252.

[17] M. Sitti and H. Hashimoto, Two-Dimensional Fine Particle Positioning Under Optical Microscope Using a Piezoresistive Cantilever as a Manipulator, Journal of Micromechatronics, 1(1), 2000, 25-48.

[18] A. Kawaji, F. Arai and T. Fukuda, 3D calibration for micro-manipulation with precise position measurement, Journal of Micromechatronics, 1(2), 2001, 117-130.

[19] M. Ammi, V. Fremont and A. Ferreira, Flexible Microscope Calibration using Virtual Pattern for 3D Telemicromanipulation, IEEE International Conference on Robotics and Automation, Barcelona (Spain), 2005, 38993904.

[20] O.D. Faugeras, Q.T. Luong and T. Papadopoulo (eds.),The Geometry of Multiple Images (MIT Press, Cambridge, Massachusetts, 2001).

[21] Mitutoyo, Microscope Units, Technical Catalog, 2004.

[22] B. McCullagh and F. Shevlin, Coplanar Camera Calibration with Small Depth of Field Lens, Irish Machine Vision and Image Processing 2004, 2004, 129-134.

[23] B. Potsaid, Y. Bellouard, and J. T. Wen, Design of an Adaptative Scanning Optical Microscope for Simultaneous Large Field of View and High Resolution, IEEE International Conference on Robotics and Automation, 2005, 460-465.

[24] M. Boissenin, et al., Computer Vision Methods for Optical Microscopes, Image and Vision Computing, 25(7), 2007, 1107-1116.

[25] G. Yang and B. J. Nelson, Wavelet-Based Autofocusing and Unsupervised Segmentation of Microscopic Images, IEEE/RSJ International Conference on Intelligent Robots and Systems, 2003, 2143-2148.

[26] J. Martin and J. L. Crowley, Experimental comparison of correlation techniques, International Conference on Intelligent Autonomous Systems, 1995, 287294.

[27] G. H. Golub and C. F. van Loan, Matrix Computation, 2nd edn., The John Hopkins University Press, 1989.

[28] J. Wu and J. Chu, Microscope Self-calibration Technique for Telemicromanipulation System, International Workshops on Microfactories, 2006.

[29] A. Shacklock, Multiple-View Multiple-Scale Navigation for MicroAssembly, IEEE International Conference on Robotics and automation, 2004, 902-907.

[30] K. Kanatani, Optimal Homography Computation with a Reliability Measure, Workshop on Machine Vision Applications, 1998, 17-19.

[31] M. A. Fischler and R. C. Bolles, Random sample consensus: A paradigm for model fitting with applications to image analysis and automated cartography, Comm. of the ACM, 24, 1981, 381-395.

[32] B. Triggs, Autocalibration and the Absolute Quadric, IEEE Conference on Computer Vision and Pattern Recognition, 1997, 609-614.

[33] P. E. Gill and W. Murray, Algorithms for the solution of the nonlinear least-squares problem, SIAM J. Numer. Anal.,15(5), 1978, 977-992. 
TABLE I

PARAMETER CALCULATION FOR DIFFERENT POSITIONS OF THE OBJECTIVE AND TUBE LENS. $T_{x}, T_{y}, E_{\text {mean }}, E_{\max }$ AND $\sigma$ ARE IN $\mu m, T_{z}$ IN $m$ AND $\alpha$ IN RADIAN.

\begin{tabular}{|c||c|c|c|c|c|c|c|c|}
\hline \multicolumn{4}{|c||}{} & \multicolumn{4}{c|}{ objective $\times 2$} & \multicolumn{4}{c|}{ objective $\times 20$} \\
\hline & \multicolumn{2}{|c|}{ Tube lens $\times 1$} & \multicolumn{2}{c|}{ Tube lens $\times 2$} & \multicolumn{2}{c|}{ Tube lens $\times 1$} & \multicolumn{2}{c|}{ Tube lens $\times 2$} \\
\hline & Proposed & Tsai & Proposed & Tsai & Proposed & Tsai & Proposed & Tsai \\
\hline \hline M & $\mathbf{2 . 1 4 3}$ & 2.146 & $\mathbf{4 . 0 4 1}$ & 4.045 & $\mathbf{2 0 . 8 5 2}$ & 20.843 & $\mathbf{3 9 . 1 3 5 3}$ & 39.136 \\
\hline$T_{x}$ & $\mathbf{- 5 5 4 . 0 3}$ & -554.52 & $\mathbf{- 2 8 0 . 8 4}$ & -281.47 & $\mathbf{- 7 2 . 1 2}$ & -71.92 & $\mathbf{- 3 8 . 5 3}$ & -38.11 \\
\hline$T_{y}$ & $\mathbf{3 2 4 . 1 2}$ & 322.25 & $\mathbf{9 7 . 7 6}$ & 97.44 & $\mathbf{- 2 8 . 2 4}$ & -28.68 & $\mathbf{- 1 4 . 9 5}$ & -15.55 \\
\hline$T_{z}$ & $\mathbf{0 . 1 4 6 3 3}$ & 0.14645 & $\mathbf{0 . 1 2 4 7 7}$ & 0.12463 & $\mathbf{0 . 1 0 4 7 8 5}$ & 0.104744 & $\mathbf{0 . 1 0 2 5 3}$ & 0.10253 \\
\hline$\alpha$ & $\mathbf{0 . 0 1 3}$ & 0.022 & $\mathbf{1 . 5 4 E - 0 3}$ & $6.66 \mathrm{E}-02$ & $\mathbf{3 . 4 8 E - 0 6}$ & $2.67 \mathrm{E}-03$ & $\mathbf{2 . 3 9 E - 0 9}$ & $2.56 \mathrm{E}-04$ \\
\hline$k_{1}$ & $\mathbf{- 3 . 3 3 E - 1 6}$ & $-4.43 \mathrm{E}-11$ & $\mathbf{- 3 . 3 E - 1 6}$ & $-4.89 \mathrm{E}-13$ & $\mathbf{2 . 7 7 E - 9}$ & $1.55 \mathrm{E}-08$ & $\mathbf{- 4 . 2 9 E - 0 8}$ & $6.8 \mathrm{E}-11$ \\
\hline$k_{2}$ & $\mathbf{6 . 3 3 E - 1 3}$ & $/$ & $\mathbf{4 . 6 3 E - 1 5}$ & $/$ & $\mathbf{- 1 0 . 9 2 E - 1 2}$ & $/$ & $\mathbf{5 . 7 9 E}-20$ & $/$ \\
\hline \hline$E_{\text {mean }}$ & $\mathbf{0 . 2 3 4}$ & 0.667 & $\mathbf{8 . 3 4 E - 0 3}$ & $5.21 \mathrm{E}-02$ & $\mathbf{2 . 5 5 E - 0 3}$ & $3.33 \mathrm{E}-02$ & $\mathbf{7 . 7 0 E - 0 4}$ & $8.64 \mathrm{E}-03$ \\
\hline$\sigma$ & $\mathbf{0 . 1 5 6}$ & 0.274 & $\mathbf{4 . 0 8 E - 3}$ & $1.08 \mathrm{E}-02$ & $\mathbf{2 . 5 4 E - 0 3}$ & $3.23 \mathrm{E}-02$ & $\mathbf{4 . 4 6 E}-05$ & $4.67 \mathrm{E}-03$ \\
\hline$E_{\text {max }}$ & $\mathbf{1 . 5 4 0}$ & 1.326 & $\mathbf{0 . 1 2 6}$ & 0.311 & $\mathbf{2 . 5 6 E - 0 2}$ & $1.78 \mathrm{E}-02$ & $\mathbf{9 . 4 9 E}-03$ & $3.55 \mathrm{E}-03$ \\
\hline
\end{tabular}

TABLE II

COMPARISON OF METHODS USING A REAL CALIBRATION PATTERN

\begin{tabular}{|c||c|c|c|c|c|}
\hline & Calibration Method & Pattern Method & $\begin{array}{c}\text { Pattern } \\
\text { size }\end{array}$ & $\begin{array}{c}\text { 3D reprojection } \\
\text { error }\end{array}$ & $\begin{array}{c}\text { Execution } \\
\text { time }\end{array}$ \\
\hline \hline Zhou et al.[12] & Tsai & Microfabricated square arrays & $10 \mu \mathrm{m}$ & $\begin{array}{c}0.672 \mu \mathrm{m} \\
20 \times \text { objective }\end{array}$ & $\begin{array}{c}\text { not } \\
\text { available }\end{array}$ \\
\hline Edwards et al.[3] & $\begin{array}{c}\text { Tsai with } \\
\text { zoom/focus estimation }\end{array}$ & Microscopic Real check pattern & $60 \times 45 \mathrm{~mm}$ & $0.3 \mathrm{~mm}$ & $\begin{array}{c}\text { not } \\
\text { available }\end{array}$ \\
\hline Seyfried et al.[?] & Tsai & Microscopic Real circular pattern & $420 \times 300 \mathrm{~mm}$ & $8 \mathrm{~nm}$ & $\begin{array}{c}\text { not } \\
\text { available }\end{array}$ \\
\hline
\end{tabular}

TABLE III

METHODS COMPARISON USING A VIRTUAL CALIBRATION PATTERN

\begin{tabular}{|c||c|c|c|c|c|}
\hline & Calibration Method & Pattern Method & $\begin{array}{c}\text { Positioning } \\
\text { precision }\end{array}$ & $\begin{array}{c}\text { 3D reprojection } \\
\text { error }\end{array}$ & $\begin{array}{c}\text { Execution } \\
\text { time }\end{array}$ \\
\hline \hline Proposed & $\begin{array}{c}\text { Modified } \\
\text { Zhang }\end{array}$ & $\begin{array}{c}\text { Virtual plane with } \\
\text { NCC Correlation and } \\
\text { template matching }\end{array}$ & $100 \mathrm{~nm}$ & $\begin{array}{c}0.234 \mu \mathrm{m} \\
\text { with } \times 2 \text { objective }\end{array}$ & $\begin{array}{c}0.827 \mathrm{~s} \\
\text { per pattern point }\end{array}$ \\
\hline Kawaji et al.[18] & $\begin{array}{c}\text { Modified } \\
\text { Tsai }\end{array}$ & $\begin{array}{c}\text { Virtual with Local Illumination } \\
\text { glass pipette tip edge detection }\end{array}$ & $0.12 \mu \mathrm{m}$ & $\begin{array}{c}0.281 \mu \mathrm{m} \\
\text { with } \times 10 \text { objective }\end{array}$ & $\begin{array}{c}\text { not } \\
\text { available }\end{array}$ \\
\hline Sitti et al.[17] & Tsai & $\begin{array}{c}\text { Virtual pattern through } \\
1 \mu \mathrm{m} \text { particle tracking }\end{array}$ & $10 \mathrm{~nm}$ & $\begin{array}{c}0.330 \mu \mathrm{m} \\
\text { with } \times 80 \text { objective }\end{array}$ & $\begin{array}{c}3 \mathrm{~s} \text { for } 5 \mu \mathrm{m} \\
\text { pushing distance }\end{array}$ \\
\hline
\end{tabular}

